\title{
Pubic Hair
}

National Cancer Institute

\section{Source}

National Cancer Institute. Pubic Hair. NCI Thesaurus. Code C33424.

Hair growing in the pubic area. 